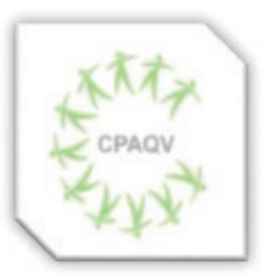

ISSN: 2178-7514
ARTIGO DE REVISÃO

\section{O DELIRIUM EM UNIDADES DE TERAPIA INTENSIVA: CONSIDERAÇÕES ACERCA DO MANEJO}

\section{Delirium in intensive care units: management considerations.}

Mayckow Carvalho da Silva Oliveira ${ }^{1}$, Bruno da Silva Lourenço ${ }^{2}$, Janaina Rosa Lourenço da Silva ${ }^{3}$, Elizeu Adriano Rocha da Silva ${ }^{4}$, Márcia Cristina Marques Pereira da Silva ${ }^{5}$, Anderson Costa Botelho ${ }^{6}$

Vol. 13| No. 2| Ano 2021

\title{
RESUMO
}

Objetivos: Conhecer as causas e fatores de risco para o delirium em pacientes internados em UTI; Evidenciar os fatores diagnósticos para o delirium em pacientes internados em UTI; Apontar as ações de assistência de enfermagem para pacientes de UTI com delirium. Metodologia: Trata-se de uma revisão bibliográfica utilizando como base as melhores evidências disponíveis na literatura científica. Resultados: Dentro da proposta estabelecida foram identificadas 20 publicações. O delirium é uma síndrome, de início agudo, com sintomas de alterações comportamentais, de sono e déficits cognitivos. É considerada uma emergência médica neuropsiquiátrica, podendo ser classificada quanto ao tempo de evolução e subtipos motores. Existem inúmeros fatores que podem levar à ocorrência de delirium, tanto predisponentes, quanto precipitantes. No ambiente de UTI existem condições específicas que favorecem ao surgimento dessa condição, com destaque para o nível de sedação e a ventilação mecânica. Conclusão: $\mathrm{O}$ tratamento para o delirium em pacientes de UTI acontece principalmente com medicamentos antipsicóticos. Evidenciou-se, também, o uso de estratégias para prevenção ou minimização do delirium, envolvendo aspectos do ambiente ou de assistência ao paciente. Nesse último caso, as ações estão mais voltadas para a enfermagem.

Palavras-chave: Delirium, Unidades de Terapia Intensiva, Enfermagem.

\begin{abstract}
Objectives: To know the causes and risk factors for delirium in ICU patients; Show diagnostic factors for delirium in patients admitted to the ICU; Point out nursing care actions for ICU patients with delirium. Methodology: This is a literature review based on the best evidence available in the scientific literature. Results: Within the established proposal, 20 publications were identified. Delirium is an acute onset syndrome with symptoms of behavioral changes, sleep and cognitive deficits. It is considered a neuropsychiatric medical emergency and can be classified according to evolution time and motor subtypes. There are numerous factors that can lead to the occurrence of delirium, both predisposing and precipitating. In the ICU environment, there are specific conditions that favor the emergence of this condition, especially the level of sedation and mechanical ventilation. Conclusion: The treatment for delirium in ICU patients happens mainly with antipsychotic drugs. The use of strategies to prevent or minimize delirium was also evidenced, involving aspects of the environment or patient care. In the latter case, the actions are more focused on nursing.
\end{abstract}

Keywords: Delirium, Intensive Care Units, Nursing.

Autor de correspondência

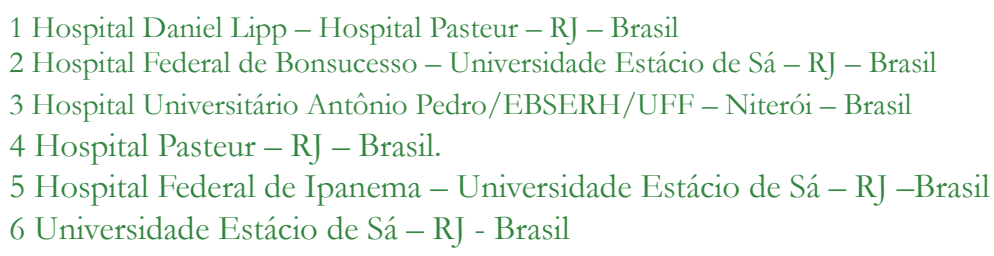

Bruno da Silva Lourenço - bru.lou@hotmail.com 


\section{INTRODUÇÃO}

O delirium é uma manifestação neuropsiquiátrica que afeta a consciência e está acompanhada por distúrbios para o estado de despertar. Ocorre com frequência em pacientes graves internados em Unidade de Terapia Intensiva (UTI) ${ }^{1}$.

O uso desse termo foi validado pelo Diagnostical and Statistic Manual of Mental Disordes, Forth Edition (DSM IV), substituindo o uso de expressões como confusão, estado mental de confusão ou psicose $e^{2}$. O ambiente de UTI, por si só, já é estressor. Sua tecnologia e frieza, aliadas às condições clínicas dos pacientes que ali se encontram, representam fator de risco para o surgimento de transtornos psiquiátricos ${ }^{3}$.

O delirium é de alta incidência em UTI. Estudos apontam que até $80 \%$ dos pacientes, variando segundo a população pesquisada, podem ser acometidos por essa manifestação, especialmente se estiverem fazendo uso de ventilação mecânica. Entretanto, somente cerca de 60\% costumam ser diagnosticados corretamente ${ }^{4}$.

O subdiagnóstico pode ser atribuído a diversos fatores e gravidade do paciente, a compreensão da equipe de saúde sobre o delirium, os instrumentos utilizados para realizar o diagnóstico e as variações de sintomas individuais são algumas das variáveis que podem contribuir para a detecção inadequada do delirium em paciente em UTI. Pacientes com delirium tendem a apresentar piores desfechos, especialmente se estiverem em estado grave. Sobre o tema, considera-se que eventualmente podem prolongar a internação hospitalar, e assim comprometer de forma grave sua recuperação global e resultar em sequelas neurológicas, que impactam diretamente na qualidade de vida do indivíduo ${ }^{5}$.

Apesar da alta incidência do delirium em pacientes de UTI, e das consequências dessa manifestação, ela tem recebido pouca atenção da equipe intensivista, especialmente porque não é a razão primária da internação ${ }^{6}$. Outro fator preocupante desse contexto é o subdiagnóstico. O não reconhecimento da patologia ou de uma manifestação clínica impossibilita a adoção de medidas eficazes para o tratamento, o que compromete, em essência, a saúde e o bom prognóstico do paciente ${ }^{7}$.

O objetivo geral desta pesquisa foi abordar o delirium em pacientes internados em UTI e os específicos: Conhecer as causas e fatores de risco para o delirium em pacientes internados em UTI; Evidenciar os fatores diagnósticos para o delirium em pacientes internados em UTI; Apontar as ações de assistência de enfermagem para pacientes de UTI com delirium.

\section{MÉTODOS}

Trata-se de uma pesquisa bibliográfica que permite ao pesquisador ter contato direto com tudo que já foi descrito, dito ou filmado, 
sobre determinado assunto, o que não seria possível se ele fosse a campo efetuar sua própria pesquisa. Esse método não é mera repetição do material existente, uma vez que propicia novo exame sobre o tema, a partir de um novo enfoque ou nova abordagem, chegando, também, a novas conclusões.

A conduta metodológica adotada foi a da pesquisa integrativa, seguindo-se os passos: a elaboração da questão norteadora; busca ou amostragem na literatura; coleta de dados; análise crítica dos estudos incluídos; discussão dos resultados e apresentação dos resultados da pesquisa .

Foram priorizados, durante a pesquisa dos dados, os meios eletrônicos que representam atualmente a democratização do acesso às informações e possibilidade de atualização permanente. Foram realizadas buscas nas bases de dados da Biblioteca Virtual de Saúde (BVS), a Scientific Eletronic Library Online (SCIELO), no Google Acadêmico, além das bibliotecas virtuais de instituições de ensino superior brasileiras.

Estabeleceu-se como critério inicial de seleção dos materiais: publicações em língua portuguesa, com texto completo, que abordassem em seu conteúdo a temática proposta, ou seja, delirium em pacientes internados em Unidades de Terapia Intensiva.

Dentro dessa proposta foramidentificadas 20 publicações, sendo uma tese, duas publicações institucionais e 17 artigos científicos que tratavam da temática proposta.

\section{RESULTADOS}

Os resultados da pesquisa foram apresentados a partir de cinco tópicos, sendo eles: (1) conceito e classificações do delirium; (2) causas e fatores de risco; (3) sintomas e critérios para diagnóstico; (4) tratamento; (5) atuação da enfermagem.

\subsection{Conceito e classificações do}

\section{delirium}

Delirium é um termo de origem latina, derivada de deliro-delirare, que corresponde a estar fora do lugar. Hipócrates, 500 anos a.C. já tratava o delirium como uma síndrome, de início agudo, com sintomas de alterações comportamentais, de sono e déficits $\operatorname{cognitivos}^{10}$.

O conceito mais aceito de delirium é o da American Pshychiatric Association (APA), de 1994, que trata que:

Delirium é uma alteração cognitiva definida por início agudo, curso flutuante, distúrbio de consciência, atenção, orientação, memória, pensamento, percepção e comportamento ${ }^{11}$.

O delirium pode ser classificado como hiperativo, hipoativo e misto $^{10}$. Delirium hiperativo é o menos comum das formas possíveis de delirium. Ocorre em paciente etilistas ou que fazem uso de sedação excessiva. É o mais fácil de ser identificado, já que seus principais sintomas envolvem aumento da atividade motora e agitação. O delirium hipoativo é aquele em que há redução do nível de consciência, apatia, letargia, 
lentificação psicomotora e redução da capacidade de resposta a estímulos externos. Já o delirium misto envolve a apresentação de características dos dois tipos anteriores ${ }^{12}$.

O delirium também pode ser classificado quanto ao tempo de evolução e subtipos motores. Segundo o tempo de evolução, o delirium pode ser prevalente, quando é detectado no momento da admissão do paciente; incidente, quando ocorre durante a internação; ou ainda, persistente, que é aquela manifestação que persiste por um período de tempo, geralmente 10 ou mais dias após o diagnóstico ${ }^{12}$.

\subsection{Causas e fatores de risco}

A fisiopatologia do delirium ainda não foi claramente identificada, mas acredita-se que esteja relacionada com diferentes mecanismos patogênicos, em seu curso final, o que leva à redução do metabolismo oxidativo cerebral e à falência da transmissão colinérgica. Além disso, a principal hipótese para o desenvolvimento do delirium está ligada a alterações nos neurotransmissores, inflamação e estresse crônico ${ }^{10}$.

Alguns fatores de risco são importantes para o aparecimento do delirium. Eles estão expostos na figura a seguir:

Figura 01 - Fatores de risco para o aparecimento do delirium ${ }^{10}$.

\begin{tabular}{|c|c|}
\hline \multicolumn{2}{|c|}{ Fatores precipitantes - Delirium } \\
\hline Medicações & Mudanças de ambiente \\
\hline Procedimentos médicos/cirurgias; & latrogenia \\
\hline $\begin{array}{l}\text { Doenças agudas: infecções, infarto } \\
\text { agudo, acidente vascular cerebral, } \\
\text { Trauma; }\end{array}$ & $\begin{array}{l}\text { Distúrbios metabólicos e } \\
\text { hidroeletrolíticos }\end{array}$ \\
\hline Imobilização prolongada & $\begin{array}{c}\text { Abuso ou abstinência de } \\
\text { substâncias (álcool, drogas } \\
\text { ilícitas) }\end{array}$ \\
\hline $\begin{array}{l}\text { Uso de equipamentos invasivos: sonda } \\
\text { vesical, sonda nasoenteral }\end{array}$ & Privação de sono prolongada \\
\hline Restrição física & Desidratação e Desnutrição \\
\hline \multicolumn{2}{|c|}{ Fatores predisponentes - Delirium } \\
\hline Déficit cognitivo pré-existente/demência & Idade maior que 65 anos \\
\hline Episódio prévio de delirium & Múltiplas comorbidades \\
\hline Doenças crônicas & Status funcional ruim \\
\hline Polifarmácia & Idade avançada \\
\hline Hepatopatia & Insuficiência renal crônica \\
\hline Doença terminal & Desidratação \\
\hline Depressão e Déficits sensoriais & Desnutrição \\
\hline
\end{tabular}

A figura 1 acima mostra que são vários os fatores que podem desencadear o delirium em paciente, com destaque para idade, medicações e condições de saúde, como a realização de procedimentos cirúrgicos. No ambiente da seguir:

UTI destacam-se a existência de pacientes com doenças agudas, com imobilização prolongada e restrição física, uso de equipamentos invasivo e mudança de ambiente, por exemplo.

Além desses fatores, a figura 2 aponta que existem diversos medicamentos que podem desencadear o delirium, conforme quadro a 
Figura 02 - Principais medicamentos desencadeantes do delirium ${ }^{10}$

Medicações associadas ao desenvolvimento de Delirium

- Anticolinérgicos: anti-histamínicos, atropina, hioscina, difenidramina, tricíclicos.

- Antimicrobianos: quinolonas, aciclovir, macrolídeos, sulfonamidas, cefalosporinas, aminoglicosídeos, anfotericina.

- Analgésicos: opióides, AINEs.

- Corticosteróides.

- Agonistas dopaminérgicos: levodopa, pramipexol, bromocriptina, amantadina.

- Anticonvulsivantes: ácido valpróico, fenitoína, carbamazepina.

- Antidepressivos: mirtazapina, inibidores de receptação de serotonina, tricíclicos.

- Sedativos: benzodiazepínicos, barbitúricos.

- Relaxantes musculares.

- Cardiovasculares: antiarrítmicos, betabloqueadores, metildopa, clonidina, diuréticos, digitálicos.

- Gastrointestinais: bloqueadores H2, metoclopramida, Ioperamida, antiespasmódicos.

- Outros: fitoterápicos, lítio, donepezil, fenotiazinas.

Observa-se, portanto, que existem inúmeras causas e fatores de risco para o delirium. E dentro do ambiente de UTI, outras condições podem representar fatores significativos para desencadeamento do delirium. $O$ ambiente desconhecido, a sedação, ruídos e alarmes constantes, mudanças contínuas de profissionais que prestam assistência e procedimentos mal explicados para os pacientes representam a maioria deles ${ }^{12}$.

A ventilação mecânica (VM) em pacientes de UTI é fator importante para o aparecimento do delirium, especialmente naqueles com quadros mais graves ${ }^{13}$. Juntamente com esse risco, destacam que pacientes submetidos à VM em UTI acabam mais sujeitos à mortabilidade (19\% a $63 \%$ ) do que pacientes em VM sem delirium $(6 \% \text { a } 32,5 \%)^{14}$.

\subsection{Sintomas e critérios para} diagnóstico

O delirium tem como sinal básico o rebaixamento do nível de consciência do indivíduo. Sinais de sonolência, mudanças no estado de alerta ou de atenção, dificuldades de concentração, distração e dificuldades de percepção de si e do meio, desorientação no tempo e no espaço também são sinais presentes e importantes para o diagnóstico do delirium ${ }^{15}$.

O paciente com delirium também mostra-se confuso, sem pensamento lógico ou pouco compreensível. Alterações na linguagem, percepção sensorial, ilusões ou alucinações visuais e/ou táteis também são comuns. Além disso, o paciente pode apresentar grande ansiedade, ideação e paranoia. Labilidade afetiva, inversão do ciclo sono-vigília compõem ainda o quadro $^{16}$.

O diagnóstico do delirium é basicamente clínico, com evidenciação de sinais e sintomas 
sugestivos da manifestação. Como não existem testes laboratoriais específicos para diagnosticálo, é necessário haver uma avaliação neurológica. Entretanto, mesmo nesses casos, o delirium é subdiagnosticado em $50 \%$ dos $\operatorname{casos}^{16}$.

Visando minimizar o subdiagnóstico tem sido adotados critérios para diagnóstico efetivo. Um dos mais utilizados fundamenta-se na Classificação Internacional de Doenças - 10 edição (CID-10) ${ }^{17}$ :

\section{a) Comprometimento}

da consciência e atenção: em um continuum de obnubilação ao coma; capacidade reduzida de focar, sustentar e mudar a atenção;

b) Perturbação global da cognição: distorções perceptivas, ilusões e alucinações; comprometimento do pensamento abstrato e compreensão, com algum grau de incoerência, comprometimento da memória imediata e recente, mas a memória remota está preservada; desorientação temporal;

c) Perturbações psicomotoras: envolvendo hipo ou hiperatividade, além de mudanças imprevisíveis; tempo de reação aumentado; aumento ou diminuição do fluxo da fala; intensificação do susto;

d) Perturbação do ciclo vigília-sono: insônia, podendo envolver perda toda do sono; sonolência diurna; piora noturna dos sintomas; pesadelos e alucinações pós despertar;

e) Perturbações emocionais: depressão, ansiedade, medo, irritabilidade, euforia, apatia ou perplexidade.
Buscando ampliar a acurácia do diagnóstico do delirium alguns instrumentos tem sido desenvolvidos e validados. Três exemplos são18:

(a) Confusion Assessment Method (CAM), baseado em características clínicas consideradas importantes para o diagnóstico, e já traduzido para o português;

(b) CAM voltado exclusivamente para pacientes em UTI, que permite avaliar pacientes que não podem se comunicar/falar, tais como os em utilização em VM;

(c) Intensive Care Delirium Screening Checklist (ICDSC) fundamentado nos critérios diagnósticos do delirium. Considerado de rápida e fácil aplicação, mas sua eficiência é mais baixo que a do CAM.

Dentre os exames disponíveis, o eletroencefalograma (EEG) é um exame utilizado para aumentar a acurácia do diagnóstico do delirium, bem como auxiliar no diagnóstico diferencial para outras condições que apresentam sintomatologia semelhante. As anormalidades encontradas no EEG que indicam delirium são: alentecimento relativo do ritmo posterior de vigília; alentecimento difuso da atividade elétrica cerebral; e atividade delta rítmica intermitente frontal ${ }^{18}$.

\subsection{Tratamento}

Considerando-se que o delirium em pacientes de UTI é condição importante e que pode atacaraté $80 \%$ dos pacientes daquela unidade, 
além de considerar que há piores desfechos para esses indivíduos, torna-se fundamental haver estratégias eficientes para seu tratamento. Uma vez que essa condição está relacionada a um desequilíbrio de neurotransmissores, há uma preferência do corpo médico pelo tratamento medicamentoso sintomático com a utilização de drogas antipsicóticas, visando uma resolução rápida da situação ${ }^{19}$.

A droga haloperidol, usada desde a década de 1970, foi uma das primeiras a serem utilizadas no caso de delirum. É considerada um antipsicótico tradicional, que age sobre a transmissão dopaminérgica no cérebro. Outros fármacos também têm sido considerados tradicionais no tratamento do delirium em UTI, tais como a clorpromazina, primozida, trifluoperazina e sulpirida. Drogas mais recentes costumam interferir nas vias serotoninérgicas, como a clozapina, olanzapina, quetiapina e risperidona. Os fármacos mais modernos costumam proporcionar os mesmos efeitos dos fármacos tradicionais, mas com uma quantidade menor de efeitos colaterais ${ }^{19}$.

Observou-se pela literatura que o tratamento padrão para o delirium é o medicamentoso sintomático. Entretanto, observou-se, uma preocupação com a abordagem de intervenção viáveis para evitar a ocorrência ou agravamento do delirium, por meio de atuação junto às variáveis modificáveis. Para esclarecimento, são variáveis não modificáveis aquelas relacionadas às características intrínsecas dos pacientes, tais como gênero, idade, tabagismo, alcoolismo, doenças prévias, demência, e/ou outras comorbidades 5 .

Assim, evidenciou, ainda nos anos de 1990, que criar um ambiente com percepções de dia/noite, janelas, utilização de luz natural, minimização das intervenções e uso de equipamentos e cateteres, ações para reduzir a privação do sono, mobilização precoce, bem como o uso de próteses como óculos ou aparelhos auditivos que estimulem o paciente, mostraram-se eficientes na prevenção e redução do agravamento do delirium ${ }^{20}$.

Além disso, boa prática clínica envolvendo controle da dor, evitar sedação excessiva, bem como escolher adequadamente o tipo de sedação a utilizar também pode influenciar no delirium e sua evolução. Os benzoazepínicos estão associados a maior ocorrência de delirium, devendo ser evitado na medida do possível $^{21}$.

\subsection{Atuação da Enfermagem}

As ações de enfermagem em relação ao delirium de paciente em UTI estiveram relacionadas com a prevenção do delirium, o que envolve ações relacionadas ao ambiente; mobilização; higiene do sono; equipamento e dispositivos; família e amigos e controle fisiológico ${ }^{22}$.

Segundo as diretrizes para o delirium da National Institute for Health and Care Excelence (NICE), as atividades de enfermagem voltadas para a prevenção do delirium em pacientes de 
UTI estão direcionadas para ações que melhorem as condições do sono (e evitem interrupções desnecessárias) e de orientação do paciente, oferta de estímulos cognitivos, facilidade para acesso de familiares, mobilização precoce e manejo da dor. Essas ações representam fatores impeditivos para o desenvolvimento do delirium, já que contribui para reduzir as variáveis que podem intensificar o quadro ${ }^{22}$.

Ainda em relação à atuação da enfermagem no ambiente de UTI, junto a pacientes com delirium, evidencia-se outras questões que podem ser praticadas pela enfermagem visando prevenir ou minimizar o delirium em curso. Os autores apontaram que minimização dos ruídos e luminosidade noturna na unidade e aplicação de escalas para detecção do delirium para detecção precoce da condição são importantes ${ }^{23}$.

É importante que a equipe de Enfermagem tenha um olhar diferenciado para a perfeita identificação do delirium, em especial o enfermeiro, que realiza a maior parte dos cuidados e está diretamente ligado à assistência que é prestada aos pacientes críticos enfermos ${ }^{24}$.

\section{CONSIDERAÇÕES FINAIS}

Odeliriuméumasíndrome, deinícioagudo, com sintomas de alterações comportamentais, de sono e déficits cognitivos. É considerada uma emergência médica neuropsiquiátrica, podendo ser classificada quanto ao tempo de evolução e subtipos motores.
Observou-se que existem inúmeros fatores que podem levar à ocorrência de delirium, tanto predisponentes, quanto precipitantes. Além disso, medicamentos também podem contribuir para o quadro. No ambiente de UTI existem condições específicas que favorecem ao surgimento dessa condição, com destaque para o nível de sedação e a ventilação mecânica.

O principal sinal do delirium é o rebaixamento do nível de consciência do indivíduo. Entretanto outros mais importantes podem surgir, como mudanças no estado de alerta ou de atenção e desorientação no tempo e no espaço, que podem variar ao longo do tempo, com piora noturna.

O diagnóstico é basicamente clínica, sendo adequado utilizar-se de instrumentos de diagnóstico e a Classificação Internacional de Doenças. O exame de eletroneuromiografia contribui para a acurácia do diagnóstico e exclusão de outras patologias.

Observou-se que o tratamento para o delirium em pacientes de UTI acontece principalmente com medicamentos para tratamento dos sintomas. Drogas antipsicóticas tem destaque nesse contexto. Além da medicação, alguns autores reportam o uso de estratégias para prevenção ou minimização do delirium, envolvendo aspectos do ambiente ou de assistência ao paciente. Nesse último caso, as ações estão mais voltadas para a enfermagem, que podem fazer o diferencial no diagnóstico precoce e no tratamento dispensado. 


\section{REFERÊNCIAS}

1. FERRAZ, A. C. et al. Estados confusionais. In: KNOBEL, E. Condutas no paciente grave. 3 ed. São Paulo: Atheneu, 2006. p. 829-45.

2. LEFEVRE, F.; LEFEVRE, A. M. C. Depoimentos e discursos: uma

proposta de análise em pesquisa social. Brasilia: Liber Livro, 2005. LIMA, D. M. O Delirium na Unidade de Terapia Intensiva. In: Santos,

F. S. Delirium: uma síndrome mental orgânica. São Paulo: Atheneu,

2008. p. 61-76.

3. SASSERON, A. B. et al. A dor interfere na função respiratória após cirurgias cardíacas? Revista Brasileira de Cirurgia Cardiovascular, v. 24, n. 04, p. 490-496, 2009. 4. ELY, E. W. et al. Delirium como preditor de mortalidade em pacientes ventilados mecanicamente na unidade de terapia intensiva. JAMA, v. 291, p.1753-62, 2004.

5. PITROWSKY, M.T. et al. Importância da monitorização do delirium na unidade de terapia intensiva. Revista Brasileira de Terapia Intensiva, v. 22, n. 3, p. 274-279, 2010.

6. PESSOA, F. P; NÁCUL, F.E Delirium em pacientes críticos. Revista Brasileira de Terapia Intensiva, v.18, n.2, p.190-5, 2006.

7. TANAKA, L. M. S. et al. Delirium em pacientes na unidade de terapia intensiva submetidos à ventilação não invasiva: um inquérito multinacional. Revista Brasileira de Terapia Intensiva, v. 27, n. 04, p. 360-368, 2015.

8. MARCONI, M. A.; LAKATOS, E. M. Fundamentos de metodologia científica. 7 ed. São Paulo/SP: Atlas, 2010.

9. SOUZA, M. T.; SILVA, M. D.; CARVALHO, R. Revisão integrativa: o que é e como fazer. Revista Einstein, v. 8, n. 1, p. 102-106, 2010.

10. LOBO, R. R.; SILVA FILHO, S. R. B.; LIMA, N. K. C.; FERRIOLli, E.; MORIGUTI, J. C. Delirium. Medicina, Ribeirão Preto, v. 43, n. 03, p. 249-257, 2010. 11. AMERICAN PSYCHIATRIC ASSOCIATION. Diagnostic and Statistical Manual of Mental Disorders, 4th edition. Washington, DC: American Psychiatric Association, 1994.

12. NELSON, L. S. A equipe de enfermagem encaminha o CAM-UTI para a triagem do delírio. Critical Care Nursing Quarterly, v. 32, n. 2, p. 137-143, 2009.

13. RIBEIRO, S. C. L.; NASCIMENTO, E. R. P.; LAZZARI, D. D.; JUNG, W.; BOES, A. A.; BERTONCELLO, K. C. Conhecimento de enfermeiros sobre delirium no paciente crítico: discurso do sujeito coletivo. Texto \& Contexto - Enfermagem, v. 24, n. 02, p. 513-520, 2015.

14. MORI, S.; KASHIBA, K. I,; SILVA, D. V.; ZANEI, S. S. SV.; WHITAKER, I. Y. Confusion assessment method para analisar delirium em unidade de terapia intensiva: revisão de literatura. Revista Brasileira de Terapia Intensiva, v. 21, n. 01, p. 58-64, 2009.

15. BRANDÃO, F. R. Delirium: um estudo comparativo entre adultos jovens, adultos e idosos. 2002. Dissertação (Mestrado) - Faculdade de Educação da Universidade de Campinas, Campinas, 2002.

16. DALGALARRONDO, P. Psicopatologia e semiologia dos transtornos mentais. 2 ed. São Paulo: Artmed, 2008.
17. CLASSIFICAÇÃO ESTATÍSTICA INTERNACIONAL DE DOENÇAS E PROBLEMAS RELACIONADOS À SAÚDE (CID-10). 10 ed. São Paulo: Universidade de São Paulo, 2003.

18. OLIVEIRA, F. R. A. Incidência, fatores preditores e consequências do delirium no pós-operatório de cirurgia cardíaca em idosos. 2015. 164f. Tese (Doutorado em Ciências) - Universidade de São Paulo, São Paulo, 2015. 19. PAGE, V. J.; CASARIN, A. Uso de antipsicóticos para tratamento do delírio na unidade de terapia intensiva. Revista Brasileira de Terapia Intensiva, v. 26, n. 02, p. 86-88, 2014.

20. INOYE, S. K.; BOGARDUS, S. T.; CHARPENTIER, P. A; LEO-SUMMERS, L. Uma intervenção multicomponente para prevenir o delírio em pacientes idosos hospitalizados. Journal Medicine, v. 340, n. 09, p. 669-676, 1999.

21. PANDHARIPANDE, P.; COTTON, B. A.; SHINTANI, A.; THOMPSON, J. PUN, B. T.; MORRIS, A. J. Prevalência e fatores de risco para desenvolvimento de delirium em pacientes com cirurgia e terapia intensiva em trauma intensivo. Journal Trauma, v. 65, n. 01, p. 3441, 2008.

22. PINCELLI, E. L.; WATERS, C.; HUPSEL, Z. N. Ações de enfermagem na prevenção do delirium em pacientes na Unidade de Terapia Intensiva. Arquivos de Medicina do Hospital da Faculdade de Ciências Médicas da Santa Casa de São Paulo, v. 60, p. 131-139, 2015.

23. FAUSTINO, T. N.; PEDREIRA, L. C.; FREITAS, Y. S.; SILVA, R. M. O.; AMARAL, J. B. Prevenção e monitorização do delirium no idoso: uma intervenção educativa. Revista Brasileira de Enfermagem, v. 69, n. 04, p. 725-732, ago. 2016.

24. Gois, J.; Vieira, 1; Lourenco, B.S.;Souza, D.R.S.; Valentim, L; Reis L.; Assistência de enfermagem ao paciente com delirium na unidade de terapia intensiva: uma revisão integrativa. Nursing (Säo Paulo) ; 22(257): 3214-3219, out.2019.

OBSERVAÇÃO: Os autores declaram não existir conflitos de interesse de qualquer natureza. 